\title{
tic\&société
}

Vol. 6, $\mathrm{N}^{\circ} 1$ | Second semestre 2012

Mutations du journalisme : nouveaux dispositifs, nouvelles pratiques

\section{Le ciblage comportemental, une perte de contrôle des éditeurs sur les données de l'audience}

\section{Alan OUAKRAT}

\section{OpenEdition}

\section{Journals}

Édition électronique

URL : http://journals.openedition.org/ticetsociete/1251

DOI : 10.4000/ticetsociete.1251

\section{Éditeur}

Association ARTIC

Référence électronique

Alan OUAKRAT, «Le ciblage comportemental, une perte de contrôle des éditeurs sur les données de l'audience ", tic\&société [En ligne], Vol. 6, N1 | Second semestre 2012, mis en ligne le 27 mai 2019, consulté le 10 décembre 2020. URL : http://journals.openedition.org/ticetsociete/1251 ; DOI : https:// doi.org/10.4000/ticetsociete.1251 
tic\&société - 6 (1), 2012

\title{
Le ciblage comportemental, une perte de contrôle des éditeurs sur les données de l'audience
}

\begin{abstract}
Alan OUAKRAT
Université Panthéon-Assas / Institut Français de Presse

Centre d'Analyse et de Recherche Interdisciplinaire Sur les Médias (Paris 2, IFP, CARISM)

10, rue Bréguet

75011 Paris.

alan.ouakrat@gmail.com

Alan OUAKRAT est docteur en Sciences de l'Information et de la Communication et ATER à I'Institut Français de Presse (Université PanthéonAssas, Paris II). II a suivi une formation en Sciences de l'Information et de la Communication puis a mené un doctorat en convention CIFRE au laboratoire Sociology and Economics of Networks and Services (SENSE) d'Orange Labs et au sein du Centre d'Analyse et de Recherche Interdisciplinaire sur les Médias (CARISM) de I'Institut Français de Presse. Ses recherches portent sur l'économie des médias et du numérique, plus particulièrement sur la publicité et la presse en ligne.
\end{abstract}




\title{
Le ciblage comportemental, une perte de contrôle des éditeurs sur les données de l'audience
}

\begin{abstract}
Résumé
Le suivi et le profilage des internautes est un enjeu de premier ordre pour les professionnels de l'industrie publicitaire. Cet article met en évidence la manière dont l'innovation constituée par le ciblage comportemental participe à transformer la logique de fonctionnement de l'activité publicitaire. Le ciblage comportemental seul n'a pas changé cette logique. II s'agit plutôt de la conjonction de la disponibilité de nouvelles technologies, portées par des entreprises de solutions technologiques qui ont, à un moment donné, rencontré les besoins des annonceurs et contribué à les redéfinir. L'exploitation des données de navigation des internautes est une condition première à ce ciblage.
\end{abstract}

Mots-clés : audience, presse, profilage, ciblage, comportemental, publicité, données.

\begin{abstract}
Monitoring and profiling of Internet users is an issue of first order for professionals in the advertising industry. This article highlights how innovation like behavioral targeting helps transform the operating logic of Internet advertising. It has not operated this change alone.. Rather, it is the combination of the availability of new technologies, driven by technological firms that has, at some point, met the needs of advertisers and helped to redefine them. The use of the Internet user's navigational data is a prerequisite to targeting.
\end{abstract}

Keywords : audience, press, profiling, behavioral targeting, advertising, data.

\section{Resumen}

El seguimiento y el análisis de los cibernautas constituye una cuestión de primer orden para los profesionales de la industria de la publicidad. Este artículo muestra la manera por la cual la innovación que ofrece el behaviorial targeting contribuye a 
Le ciblage comportemental,

une perte de contrôle des éditeurs sur les données de l'audience

transformar la lógica de funcionamiento de la actividad publicitaria. No obstante, esta innovación, por sí sola, no ha originado los cambios; más bien ha sido la disponibilidad de nuevas tecnologías, quienes, impulsadas por empresas que ofrecen soluciones tecnológicas, han buscado solucionar, en un determinado momento, las necesidades de los anunciantes, a la vez que dichas necesidades han sido redefinirlas. En este sentido, la explotación de los datos de navegación de los internautas es una condición básica para el citado behaviorial targeting.

Palabras claves: audiencia, prensa, perfiles, behavioral targeting, publicidad, datos. 
Le marché publicitaire a connu un élargissement sans précédent sur internet, marqué par l'entrée d'acteurs tels que les portails et les moteurs de recherche. Appartenant à des groupes issus de l'informatique et des télécommunications, ces acteurs ont constitué les plus fortes audiences de l'internet. Les moteurs de recherche et les portails sont d'abord financés par la publicité, qui est un des éléments centraux du modèle d'affaires des médias. Pour remédier au déclin d'appétence et d'attention pour la publicité en ligne, les industriels ont mis au point différents formats publicitaires et pratiqué le ciblage de la publicité. Le ciblage comportemental est basé sur la collecte et le traitement des données d'historique de navigation des internautes. II fut d'abord présenté comme un moyen de lutter contre une tendance déflationniste des prix des espaces publicitaires sur internet, liée à l'abondance de l'espace publicitaire disponible et à l'efficacité faible des bannières. Or, cette innovation paraît traduire un mouvement plus profond de l'industrie publicitaire prenant appui sur l'exploitation des traces laissées par la navigation des individus sur internet. De nouveaux entrants de l'industrie publicitaire cherchent à intermédier la vente de publicité par une proposition de ciblage comportemental des audiences. II s'agit ainsi de produire, par des inférences et des statistiques probabilistes, de la prévisibilité dans les comportements des audiences.

Notre article s'appuie sur une enquête de terrain réalisée dans le cadre de notre travail doctoral ${ }^{1}$. Nous nous intéressons plus particulièrement ici à l'innovation constituée par le ciblage comportemental pour les éditeurs de la presse en ligne sur la période 2008-2010. Notre réflexion est organisée en trois parties, articulées autour d'une question centrale: dans quelle mesure les solutions techniques de ciblage comportemental participent-elles à redessiner le fonctionnement de l'activité publicitaire sur internet? La première partie décrit le contexte d'apparition de ces techniques sur le marché français de la publicité en ligne, ainsi que les acteurs et le mode de fonctionnement du ciblage comportemental. La deuxième partie présente les transformations de l'achat média, se basant de façon croissante sur des données relatives à la navigation des internautes, et les difficultés relatives à l'exploitation de ces données. Enfin, la troisième partie analyse les enjeux et les risques de ces techniques pour les éditeurs, tout en resituant le ciblage comportemental dans un mouvement d'évolution plus général de la publicité sur internet qui tend vers la collecte et le traitement automatisé des informations sur l'audience.

\footnotetext{
${ }^{1}$ L'enquête a été menée entre le $1^{\text {er }}$ décembre 2008 et le 26 février 2010 . Elle a consisté en 39 entretiens qualitatifs semi-directifs auprès de professionnels occupant des postes à responsabilité dans le monde de la presse et de la publicité en ligne.
}

tic\&société - 6 (1), 2012 
Le ciblage comportemental,

une perte de contrôle des éditeurs sur les données de l'audience

\section{Origines et fonctionnement du ciblage comportemental}

La publicité a pour objectif l'appariement entre un message à vocation commerciale et une audience-cible (Gabzsewicz et Sonnac, 2006). Le ciblage permet aux entreprises d'éliminer le "gaspillage " dans les investissements publicitaires, lorsque les préférences des consommateurs ne correspondent pas aux caractéristiques des produits. S'assurer que la publicité est achetée de façon efficace et non dirigée vers les " mauvaises personnes » a toujours été un défi pour les marketeurs. II s'agit d'une préoccupation classique des publicitaires depuis le XIXe siècle, résumée de façon humoristique par la célèbre expression de John Wanamaker, propriétaire d'un grand magasin à Philadelphie : " La moitié de la monnaie que je dépense dans la publicité est "gaspillée" et le souci est que je ne sais pas quelle moitié " (lyer et al., 2005).

Dans un contexte où la concurrence pour les revenus publicitaires est exacerbée sur internet, l'usage des données des internautes pour enrichir le ciblage publicitaire constitue une manière de différencier leur offre pour les régies publicitaires.

La publicité en ligne se répartit, essentiellement, entre la publicité graphique (display) et les liens sponsorisés. Alors que les seconds proposent des annonces textuelles contextualisées (c'est-à-dire aux côtés d'un contenu ou de résultats de recherche), la première développe une multitude de formats graphiques, parfois animés (rich media). Les liens sponsorisés (search) ont progressivement dominé la répartition des investissements publicitaires sur internet. La publicité graphique retient particulièrement notre attention ici, dans la mesure où elle est le format plébiscité par les annonceurs sur les sites de presse $^{2}$.

Depuis l'apparition en 1994 sur le site HotWired.com de la première bannière publicitaire, l'efficacité de ce format mesuré par le taux de clic n'a cessé de s'éroder ${ }^{3}$. Or, il s'agit du format le plus répandu de la publicité graphique. Si l'on

${ }^{2}$ Les sites de contenu sont pour l'essentiel absents du marché du marketing direct en ligne (IDATE, 2010).

Alors que le taux de clic moyen était équivalent à $7 \%$ en 1996, il n'était plus que de $0,7 \%$ en 2002 (Drèze et Hussherr, 2003). L'efficacité des bannières publicitaires, telle que mesurée par le taux de clic, a donc été divisée par dix en six ans. Des données plus récentes indiquent le prolongement de cette tendance baissière, révélant des taux de clic moyen se situant entre 0,2 et $0,3 \%$ pour les bannières traditionnelles (IDATE, 2010) ou descendant même jusqu'à $0,17 \%$ tous formats confondus (SmartAdServer, 2009). II convient néanmoins de relativiser ces résultats, la publicité pouvant avoir un objectif autre que la vente à court terme, comme le fait d'accroître la notoriété 
considère que la tâche d'une bannière est d'attirer l'attention de l'internaute pour l'amener ensuite à cliquer sur la publicité, force est de constater l'inefficience de ce format. Ce phénomène est aussi connu dans la littérature sous le nom d'« invisibilité des bannières publicitaires " (banner blindness). II serait dû à la perturbation de l'activité de l'internaute par la publicité, la sursollicitation commerciale du consommateur le conduisant à ignorer la publicité (Chang-Hoan and Hongsik, 2004). Ainsi, les internautes éviteraient de fixer leurs yeux sur ce qui pourrait s'apparenter à une bannière publicitaire, engendrant par conséquent une chute des performances mesurées en taux de clic de ces espaces publicitaires.

Pour attirer l'attention sur la publicité, les annonceurs ont d'abord mené un jeu sur le format afin de créer un "biais de visibilité, une saillance, dans un univers saturé de signaux perceptuels » (Kessous et al., 2010, p.13). L'Internet Advertising Bureau (IAB) a encadré la standardisation des formats de la publicité en ligne. À partir de 2003, des spécifications techniques ont été établies pour uniformiser l'offre et réduire les coûts de coordination entre les annonceurs et les éditeurs de sites web. Mais pour se singulariser et différencier leur offre, les régies publicitaires se sont lancées dans l'innovation de formats. Cette dernière a consisté à produire des formats plus attrayants visuellement afin de capter l'attention des internautes (Goldfarb and Tucker, 2011). L'efficacité, mesurée par le taux de clic, varie selon le format publicitaire utilisé. Cette stratégie trouve aussi ses limites dans la mesure où l'innovation de formats est rapidement imitée.

Une autre stratégie a consisté à diffuser de la publicité de façon plus ciblée, afin de rehausser les prix des espaces publicitaires commercialisés.

\subsection{Le développement du ciblage comportemental en France (2008- 2010)}

Internet permet d'accumuler un nombre important de données sur le comportement de navigation des individus (data-mining). Les annonceurs réduisent ainsi, en partie, la nuisance provoquée par une publicité non ciblée et non désirée des consommateurs. De plus, la publicité en ligne offre la possibilité de suivre les actions du prospect après son exposition à la publicité (les sites visités, les recherches effectuées, etc.). Le ciblage comportemental permet d'augmenter la pertinence de la publicité pour un individu et de créer de la rareté, certaines cibles ayant plus de valeur que d'autres d'un point de vue

d'une marque ou d'un produit. Dans ce cas, le taux de clic n'est pas un indicateur témoignant de l'efficacité, comme l'ont montré différentes études (Drèze et Hussherr, 2003 ; Hollis, 2005).

tic\&société - 6 (1), 2012 
Le ciblage comportemental,

une perte de contrôle des éditeurs sur les données de l'audience

marketing. La nouveauté avec internet consiste à s'appuyer sur des données individuelles pour adresser des publicités ciblées, indépendamment du support et du contenu de la page visitée.

Apparu aux États-Unis en 2001, le ciblage comportemental a été tout d'abord mis au point par des acteurs issus de l'internet. L'idée est de rehausser de "quelques centimes » les prix bas de la publicité sur Internet, en raison de l'abondance de l'espace publicitaire disponible. Le ciblage comportemental offre des solutions de monétisation d'un inventaire ${ }^{4}$ peu qualifié, difficile à vendre et à différencier. En France, l'implémentation de ces solutions est plus récente, et a vu son essor en 2008-2009.

Les portails ont été les premiers à intégrer ces solutions, car ils ont une audience massive à monétiser. En effet, la condition sine qua non pour parvenir à effectuer le ciblage comportemental des audiences-cibles est de couvrir une audience importante. L'usage des données comportementales des audiences permet de sortir du mode de valorisation ordinaire, de segmenter des cibles à l'intérieur de l'audience globale. Cette démarche rencontre l'appétence des annonceurs pour les données relatives à l'audience. La difficulté sur internet n'est pas tant d'apporter du contenu à l'audience que de l'audience aux contenus (Van Couvering, 2008). L'industrie des télécommunications est entrée dans la concurrence pour les revenus publicitaires, en parvenant à s'imposer dans l'univers des portails. Cependant, les sites médias qui ont essayé de répliquer cette stratégie de "course à l'audience " n'ont pas réussi « à faire le poids " et à s'imposer face à ces acteurs (Attias, 2008). Les plateformes d'audience que sont les portails et les services web sont parvenues à rassembler une audience quantitativement très importante. Ils ont ensuite revendu cette audience massive aux annonceurs. Étant donné l'incertitude liée à l'efficacité de la publicité en ligne, les annonceurs ont pu faire fortement pression sur les tarifs. La bannière publicitaire, vendue au nombre d'impressions (CPM), a vu son prix moyen s'effondrer. Pour revaloriser cet inventaire peu différencié, il a fallu créer de la rareté à partir des profils des internautes consultant ces pages web. Les techniques de ciblage comportemental ont alors été déployées, en se basant sur la collecte et l'analyse des données de navigation. Cette énorme masse d'informations fait l'objet de traitements statistiques destinés à déterminer la composition de l'audience et à valoriser des segments d'audience spécifiques auprès des annonceurs.

Le marché de la publicité sur Internet est un marché relativement jeune, où de nombreux acteurs tentent de jouer le rôle d'intermédiaires pour s'intégrer

${ }^{4}$ Ensemble des espaces publicitaires commercialisés sur un site ou un ensemble de sites web. 
dans la chaîne de valeur ${ }^{5}$. L'innovation technique est un des moyens les plus sûrs d'y parvenir. Les acteurs qui portent ces innovations, notamment celle que constitue le ciblage comportemental, sont souvent nés en ligne. Ils se basent davantage sur une culture professionnelle relative à l'ingénierie technique plutôt qu'à la publicité média traditionnelle. Les régies publicitaires des sites de presse provenant d'un monde différent, celui de la publicité média, doivent collaborer avec ces nouveaux acteurs. Ces derniers peuvent aussi bien être des start-up qui fournissent des solutions techniques de ciblage ou d'innovations de formats publicitaires, que des réseaux publicitaires destinés à requalifier ${ }^{6}$ une partie de l'inventaire, ou encore des " géants de l'Internet ", capables à la fois de donner une exposition plus grande aux contenus et d'associer à ces contenus des solutions techniques publicitaires perfectionnées de monétisation. II s'agit souvent de petites sociétés hyper spécialisées dans l'innovation technique qui, avec une visée mondiale, développent des outils de suivi des internautes. Ces solutions sont ensuite soit directement implémentées dans les processus de vente de publicité par les régies publicitaires d'acteurs internationaux, les " géants de l'Internet ", qui rachètent ces sociétés et s'approprient leurs outils, soit proposées aux régies ou aux annonceurs sous la forme de solutions techniques adaptées à leur activité.

Le ciblage comportemental vise avant tout à revaloriser des espaces peu ou pas valorisables en tant que tels. Internet obéit à des règles spécifiques sur le plan publicitaire, partiellement inscrites dans son fonctionnement technique. Toutefois, ces règles de fonctionnement de l'activité publicitaire sur internet ne se sont pas imposées d'elles-mêmes. Elles ont bénéficié de l'appui et du soutien des annonceurs insatisfaits du produit publicitaire institué et de l'activisme d'innovateurs, soucieux de proposer au marché des outils adaptés au fonctionnement du média.

La publicité sur Internet est délivrée individuellement à chaque ordinateur, en fonction de l'historique de navigation, de l'exposition préalable à la publicité de l'internaute ainsi que de ses préférences enregistrées et/ou déclarées. Les données comme les sites visités et les contenus consultés sont traçables grâce à des petites pièces d'information appelées les "cookies ${ }^{7}$ » et stockées dans

\footnotetext{
${ }^{5}$ La chaîne de valeur renvoie à l'ensemble des acteurs impliqués dans le processus de production d'un bien ou d'un service. Ce concept a été importé en économie à partir des sciences de la gestion et des écrits de Porter (1986). La chaîne de valeur permet de schématiser et de décrire l'organisation ou la réorganisation d'un secteur, d'une filière ou d'une entreprise.

Dans une perspective proche de la sociologie économique, nous considérons le travail de valorisation comme un travail d'attribution des qualités au produit publicitaire.

${ }^{7}$ Les cookies sont des petits fichiers texte placés sur l'ordinateur de l'internaute par le serveur du site ou un tiers autorisé par le site. On distingue ainsi les " cookies primaires" (first party cookie) envoyés par le domaine principal sur lequel l'internaute navigue des cookies " tiers " (third party
} 
Le ciblage comportemental,

une perte de contrôle des éditeurs sur les données de l'audience

les navigateurs. L'utilisation des cookies est plus efficace lorsque beaucoup de sites web appartiennent à un même réseau de sites pour partager l'information. L'usage des cookies requiert des internautes qu'ils ne les suppriment pas trop souvent (Johnson, 2009). Ces données sont collectées de façon passive, c'està-dire sans l'intervention de l'internaute et ne nécessitent pas l'expression d'un consentement explicite. Les informations sont récupérées de façon invisible pour l'internaute par des échanges de données entre les serveurs web et le navigateur. Elles circulent par le biais des cookies ou d'« image-pixel » (web bugs $^{8}$ ) (Goldfarb et Tucker, 2010).

Le principe du ciblage comportemental est d'adresser de la publicité aux individus, indépendamment du site et de la page visitée, uniquement en fonction de leur profil. Ce dernier est constitué à partir de l'enregistrement de diverses données individuelles, qui sont conservées entre trois et six mois ${ }^{9}$. Chaque profil individuel d'internaute est comparé à des profils-types, selon une logique de scoring, dans la mesure où il s'agit d'établir une concordance entre les données recueillies sur le comportement d'un internaute et des profils-types déterminés à l'avance. Le ciblage comportemental (ou behavioral targeting) déduit en fonction du " comportement de l'internaute ", de sa logique de navigation, le message publicitaire à lui délivrer. II s'agit notamment de partir des centres d'intérêt de l'internaute, inférés à partir des contenus qu'il consulte.

\subsection{L'extension de la chaîne de valeur par une intermédiation "technique »}

Les solutions de ciblage comportemental ont été principalement réalisées par des sociétés axées sur la proposition d'outils technologiques à destination du marché publicitaire.

Les acteurs de l'internet ont une perception transnationale de l'activité publicitaire. Issus de cet environnement, ils n'ont pas d'effort d'adaptation à faire pour s'adapter aux exigences des annonceurs qu'ils ont fortement influencées et contribuées à construire. Ils ont ainsi intégré et incorporé dès leur naissance la logique de fonctionnement du média Internet, natifs de cet environnement.

cookies) qui proviennent des domaines sur lesquels sont stockés des éléments de la page tels que des publicités, mais avec lesquels l'internaute n'a pas de relation directe et explicitement consentie. Les informations recueillies font ensuite l'objet d'une exploitation par les serveurs publicitaires qui ajustent la distribution et l'affichage de publicité en fonction de celles-ci.

Les web-bugs, aussi appelé "action tags ", "web tags » ou "beacons ", sont des mouchards invisibles qui prennent la forme d'une image minuscule, de la taille d'un pixel incrusté dans une page web. Ils sont certes affichés, mais invisibles pour l'utilisateur, à moins d'accéder au code html de la page web et de déchiffrer la ligne de code qui les définit.

Sous réserve de non effacement des cookies par l'internaute ou un logiciel-tiers. 
Le degré d'intégration des outils techniques a une incidence sur le jeu concurrentiel. Ainsi, c'est par l'innovation technique que de nouveaux acteurs parviennent à intégrer la chaîne de valeur de la publicité graphique et à intermédier les transactions. Par sa technicisation croissante, le marché de la publicité en ligne nécessite de plus en plus le recours à des experts, capables d'accompagner les acteurs à tirer parti des potentialités technologiques du média, notamment au niveau de l'analyse des données. De nouveaux entrants se sont intégrés au marché publicitaire en proposant des solutions technologiques destinées à valoriser les audiences auprès des annonceurs ${ }^{10}$. Ce mouvement a provoqué une extension de la chaîne de valeur de la publicité en ligne en renforçant le rôle d'intermédiation, à l'interface de l'offre et de la demande sur ce marché de professionnels $(B t o B)$.

Le ciblage comportemental peut prendre plusieurs formes et être plus au moins intégré à l'activité de l'entreprise qui y a recours. Lorsqu'il est externalisé, cela signifie qu'une entreprise confie une partie de son inventaire publicitaire à un réseau chargé de mettre en œuvre le ciblage avec ses propres outils, c'est le cas par exemple de Specific Media ${ }^{11}$.

Le ciblage comportemental peut aussi être internalisé, c'est le cas lorsqu'une entreprise utilise les solutions technologiques d'une entreprise-tierce lors de la vente de son propre inventaire. La société technologique agit alors comme un prestataire de services et prélève une commission sur le nombre d'impressions délivrées ayant recours à des données de ciblage comportemental. C'est le modèle de Weborama. En France, Weborama est l'une des premières sociétés à s'être lancée dans le ciblage comportemental. Entreprise française fondée en 1998 par deux docteurs en physique et un ingénieur en informatique, Weborama s'est spécialisée dans l'ingénierie technique de l'Internet. Dans un premier temps, l'entreprise produit des outils de mesures d'audience serveur

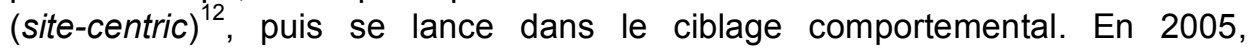
l'incubateur de start-up, StartUp Avenue, prend le contrôle de la société, et lui

\footnotetext{
${ }^{10}$ Ces dispositifs de valorisation des audiences complètent les dispositifs classiques de valorisation publicitaire de l'audience des médias, tels que la mesure et les études d'audience.

Réseau publicitaire blind, Specific Media agrège un réseau de 300 sites web. Un réseau publicitaire blind désigne une régie publicitaire externe qui agrège les espaces publicitaires de différents sites. Le terme "blind " correspond au fait que la vente de publicité sur ce réseau ne permet pas aux annonceurs de savoir sur quel site leur publicité va être affichée, ils doivent simplement composer avec l'assurance que la campagne publicitaire sera délivrée un certain nombre de fois auprès d'une audience, répondant à des caractéristiques définies entre les deux parties (en termes d'âges, de sexe, de catégorie socio-professionnelle et parfois de centres d'intérêts).

La société continue de proposer sa solution de mesure d'audience côté sites (site-centric), labellisée par l'Office de Justification de la Diffusion (OJD).
} 
Le ciblage comportemental,

une perte de contrôle des éditeurs sur les données de l'audience

permet ainsi son entrée en Bourse. Les activités de la société sont repositionnées sur le ciblage comportemental et Alain Lévy, fils de Maurice Lévy, PDG de Publicis et président de Start-Up Avenue, devient Président directeur général de Weborama. Alain Lévy, ayant suivi une formation d'ingénieur et des études au Massachusetts Institute of Technology (MIT) de Boston, constate que les ingénieurs sont absents du monde de la publicité ${ }^{13}$. Nous avons rencontré lors de notre enquête un responsable de Weborama, qui résume ainsi la proposition de valeur faite par les solutions de ciblage comportemental :

«On ne valorise pas un espace sur la valeur de son contenu, mais sur la valeur du profil qui va consulter ce contenu. » (responsable Weborama, 23/12/08)

La publicité en ligne y est considérée quasi exclusivement sous l'angle technologique. L'« approche contact » est privilégiée à une approche média: " Je vends un contact, indépendamment de l'endroit où je vais le trouver». Les entreprises de ciblage comportemental n'ont pas besoin d'avoir recours à des données « identifiantes » telles que le nom ou le numéro de sécurité sociale des individus, mais des données relatives au comportement de navigation des internautes.

"Ce sont des systèmes qui ftonctionnent sur des lois du grand nombre. Je n'ai pas besoin de suivre une personne en particulier. " (directeur général, Weborama)

Déployées sur les invendus de l'inventaire publicitaire de grands sites, les solutions de Weborama valorisent cet inventaire en fonction des profils des internautes qui consultent ces sites web. Ces profils sont répertoriés et classés en fonction de thématiques :

« Nous vendons des segments de profils sur des sites épars après avoir trouvé une cohérence au réseau de sites. Les réseaux sont une multitude de sites qui n'ont rien à voir ensemble, mais qui ont des internautes qui,

\footnotetext{
${ }^{13}$ Entretien de Jérome Bouteillier avec Alain Lévy, "Portrait d'entreprenaute », Publiée le jeudi 18 décembre 2008: http://www.clubic.com/actualite-247028-portrait-entreprenaute-alain-levyweborama.html, Consulté le 9 novembre 2012.
} 
pris dans leur ensemble, peuvent permettre de créer des segments. Ce sont ces segments que nous allons vendre. » (directeur général, Weborama)

II existe de nombreuses méthodes de constitution de profil. Par exemple, Yahoo! agrège des données relatives aux pages visitées, aux parcours, aux requêtes tapées dans le moteur de recherche, ainsi qu'aux interactions de l'utilisateur avec la publicité.

«On a des solutions marketing très poussées avec lesquelles [les annonceurs] se moquent de savoir où ils passent, mais ils veulent des choses très ciblées. On touche la bonne personne, au bon moment avec la bonne offre, mais le bon moment est un élément important. On regarde tout ce que font les internautes. Les pages médias qu'ils consultent, les requêtes search qu'ils tapent et les clics sur les liens sponsorisés ou sur les pubs. On ne va pas dans les mails, les trucs perso, tout ce qui est données [enregistrées]... » (directrice commerciale Yahoo! France, 22/01/09)

Le ciblage comportemental de la publicité en ligne n'est cependant pas une réponse appropriée pour les espaces publicitaires haut de gamme (premium). De plus, il doit encore faire les preuves de sa pertinence et de son efficacité.

\section{Enjeux et limites du ciblage comportemental}

Alors que le marché voit les données navigationnelles et comportementales comme une opportunité marketing pour cibler les campagnes publicitaires, d'autres s'interrogent quant à la propriété de ces données et à l'usage qui peut en être fait (Kessous et al., 2010). La collecte et le traitement de ces données correspondent aussi à des enjeux économiques, culturels et sociaux sousjacents.

\subsection{Une simple transformation de l'activité d'achat média ?}

L'achat média s'est complexifié et sophistiqué avec la fragmentation de l'audience des médias existants et l'apparition de nouveaux supports de publicité. Le ciblage comportemental témoigne de la transformation de l'activité d'achat média. II dessine un mouvement qui a des implications plus vastes pour 
Le ciblage comportemental,

une perte de contrôle des éditeurs sur les données de l'audience

le fonctionnement de la publicité en ligne, tel que le rapprochement de la publicité média et du marketing direct. Sur internet s'efface progressivement la frontière séparant la publicité média et les actions de communication directes dites hors-média (Turow, 2012). En tant que média, internet a la particularité d'être à la fois une place de marché publicitaire et commerciale. Cette caractéristique détermine en grande partie le rapprochement qui s'y est opéré entre publicité en ligne et marketing direct (Ouakrat et al., 2010). Ces techniques de collecte et de traitement de données des internautes participent à l'hybridation des logiques de fonctionnement de la publicité média et du marketing direct sur internet. En ajoutant une couche de données supplémentaire au commerce de publicité, le ciblage comportemental oriente les éditeurs vers un rôle de marchandisation des données comportementales de son audience.

Des spécialistes des statistiques probabilistes ont ainsi investi le marché publicitaire pour donner un sens aux données collectées sur le comportement de navigation des internautes. Ces données vont équiper les décisions d'investissement des annonceurs en matière d'achat de publicité.

Le changement de paradigme publicitaire provoqué par l'utilisation des technologies numériques de ciblage engendre une transformation de l'activité du commerce d'espaces publicitaires. On passe ainsi d'une publicité insérée aux côtés d'un contenu dans un environnement particulier au ciblage d'internautes selon des caractéristiques spécifiques. Ces deux logiques ne sont pas étrangères l'une à l'autre. II s'agissait bien à travers l'achat d'un emplacement de toucher une audience mesurée et anticipée (predicted and mesured audience) (Napoli, 2003, p.29-35). L'achat cible désormais l'audience en fonction de caractéristiques inférées par son comportement de navigation telles que ses centres d'intérêt et son profil sociodémographique. Ainsi, ces techniques profitent du fait que ce n'est pas « l'attention effectivement accordée à tel ou tel contenu » qui est commercialisée par les régies publicitaires, mais une occasion de voir, qui n'est qu'une « approximation de l'attention accordée réellement à ces contenus » (Kessous et al., 2010, p.14).

Comme toute technique, le ciblage comportemental déplace et transforme les enjeux du système qu'il contribue à faire évoluer. La technique n'est pas neutre : "Elle a son propre poids, ses propres déterminations, ses propres lois, autrement dit, en tant que système elle évolue en imposant sa logique » (Ellul, 1988, p.284). Et tout progrès technique comporte un grand nombre d'effets imprévisibles. La technologie participe à la transformation de la logique de l'activité publicitaire, mais son développement est intimement lié au contexte politique, social et économique dans lequel il se produit (Van Couvering, 2008). 


\subsection{Les limites du ciblage comportemental}

Le ciblage comportemental n'est pas pertinent pour les campagnes de publicité qui ont pour objectif la notoriété, puisqu'il s'agit alors de diffuser le message le plus largement possible. En effet, les annonceurs, en optant pour le ciblage comportemental, doivent réaliser un arbitrage entre la précision et la couverture de leur cible. Ainsi, dans une campagne de publicité qui vise la notoriété, c'est davantage une audience élargie qui va être visée plutôt que la recherche d'une vente directe en s'adressant à une cible restreinte.

"Les hommes se comportent de telle manière, ensuite toute personne qui se comporte d'une manière identique a une forte chance d'être un homme. Un annonceur, il ne veut pas toucher une personne, il veut en toucher des millions. Si vous rentrez dans le détail, vous touchez trois personnes et vous n'intéressez plus personne. Ou alors, vous faites du marketing direct. Ce n'est pas le même métier. Ce que fait Weborama, c'est du média, ce n'est pas du horsmédia. Ce qui nous intéresse nous, c'est d'avoir des informations sur le maximum de gens. Et de fait, ça restreint l'information. Plus vous allez être précis, moins vous aurez [de monde]. » (responsable Weborama)

Le ciblage comportemental est une manifestation d'un phénomène plus large lié à l'automatisation et à la personnalisation de la publicité en ligne. Les données publicitaires sont collectées à l'insu des utilisateurs, ce qui pose des problèmes liés au respect de la vie privée des internautes et la question de la propriété de ces données.

Les industries financière et publicitaire présentent des similitudes dans l'opacité de leurs pratiques pour le grand public et le régulateur. Comme l'a démontré la crise financière, les implications de ces pratiques peuvent être considérables et affecter l'ensemble de la société (Turow, 2012). En particulier, le système des places de marché publicitaire (ad-exchange) et d'achat en temps réel de la publicité (real-time bidding) sur des plateformes dédiées à cet effet et basées sur le ciblage des internautes grâce à leurs données de navigation, interroge dans son développement et ses perspectives. La boîte noire que constituent ces approches, marquées par le manque de transparence, suscite des inquiétudes et appelle un débat public. La question de la régulation de l'accès et de l'usage des données personnelles des utilisateurs par les entreprises à des fins commerciales rejoint les préoccupations actuelles de 
Le ciblage comportemental,

une perte de contrôle des éditeurs sur les données de l'audience

chercheurs sur le phénomène des «Big Data » (grands ensembles de données) (Boyd, Crawford, 2011). Les données brutes ne s'expliquent pas d'elles-mêmes. L'interprétation est au cœur de l'analyse des données. Prenant acte du fait qu'il existe " un mouvement de fond, gouvernemental et industriel, pour récolter et extraire le maximum de valeur des données ", les auteurs rappellent que la donnée la plus accessible et qui circule le plus concerne l'historique de navigation des internautes. La question d'une régulation de l'usage des données personnelles à des fins commerciales fait débat ${ }^{14}$. Jusqu'ici, les consommateurs se sont peu saisis de ces enjeux, bien qu'ils manifestent souvent une réaction négative à la publicité en ligne, comme en témoigne leur comportement d'évitement de la publicité. D'un point de vue théorique et pratique, le ciblage publicitaire est censé réduire la nuisance provoquée par une publicité non ciblée. Cependant, il n'est pas certain qu'une publicité personnalisée soit désirée par les internautes. Ils peuvent aussi la rejeter, s'ils la considèrent comme une atteinte à leur vie privée. C'est ce qu'indiquent certains des travaux de recherche consultés (Goldfarb, Tucker, 2010). L'appréciation de la publicité en ligne par les consommateurs est liée au degré d'intrusion ressenti dans leur vie privée. Il est reproché aux entreprises le manque de lisibilité sur les données collectées et l'usage qui en est fait, ainsi que la possibilité de refuser d'être suivi ${ }^{15}$. Les industriels font entendre leur voix à travers leurs instances représentatives telles que I'IAB. Ils estiment qu'une régulation du marché limiterait la capacité des sites web à suivre les consommateurs et nuirait à l'économie des entreprises qui ont recours au ciblage comportemental.

Aux États-Unis, l'initiative Do No Track a été mise en œuvre en 2011. Encouragé par la Commission fédérale du commerce (FTC) qui veille à la protection des consommateurs américains, Do No Track a été développé par des chercheurs de l'Université de Standford. II s'agit d'empêcher les annonceurs d'envoyer des publicités individualisées. Do No Track se présente sous la forme d'une extension qui peut être intégrée à la plupart des navigateurs ${ }^{16}$. Cette dernière vise à simplifier l'accord ou le refus des utilisateurs quant à l'usage de leurs données de navigation. Toutefois, les internautes doivent avoir la

\footnotetext{
${ }^{14}$ Les groupes de défense des libertés individuelles, tels que l'Electronic Frontier Foundation aux Etats-Unis, ont émis de nombreuses critiques à l'égard de ce procédé qui consiste à suivre l'utilisateur de manière intentionnellement invisible.

${ }^{15}$ Schatz, A. 2009. Regulators rethink approach to online privacy. Wall Street Journal (August 5), http://online.wsj.com/article/SB124949972905908593.html

${ }^{16}$ À l'exception du navigateur Chrome de Google.
} 
connaissance de cet outil et l'activer eux-mêmes, ce qui jusqu'ici en a grandement limité la diffusion ${ }^{17}$.

\section{L'usage des données personnelles à des fins publicitaires}

Le marché de la publicité en ligne est un marché qui se caractérise par un niveau élevé d'innovation. Le dilemme pour les éditeurs est de ne pas être trop attentiste, de peur de rater le train du progrès, ou au contraire de ne pas trop se précipiter, pour éviter d'investir trop tôt dans une solution qui ne serait pas adoptée par les principaux acteurs du marché.

Les éditeurs de presse sont engagés dans une relation asymétrique avec les annonceurs. Ces derniers bénéficient d'un pouvoir croissant face à une dépendance économique accrue des médias aux ressources publicitaires. Autant dans un marché fondé sur une concurrence limitée, un nombre d'acteurs établis, des contraintes de capacité relatives à la disponibilité de l'espace publicitaire et en situation d'oligopole, les titres de presse avaient une large marge de manœuvre face aux annonceurs. Autant dans la publicité en ligne, avec un univers de concurrence élargi, l'abondance de l'espace publicitaire disponible et la fragmentation de l'offre publicitaire, les annonceurs sont davantage en mesure de faire entendre leurs exigences. La répartition des investissements publicitaires des annonceurs conditionne la survie d'un certain nombre de sites web. La gestion de ces investissements s'appuie sur un nombre croissant de données relatives aux comportements des internautes.

\subsection{Les éditeurs de presse, cibles du ciblage comportemental}

Les éditeurs des sites de presse ont été sollicités pour réaliser le ciblage comportemental des espaces publicitaires les moins aisément valorisables sur leur site web. En dehors de leur offre "premium » liée à la vente d'espaces publicitaires en page d'accueil du site ou de rubriques spécifiques, les sites de presse disposent d'un grand nombre d'espaces plus difficilement valorisables.

Début 2009, les régies publicitaires des sites médias, à l'instar de Régie Obs., réfléchissaient à implémenter ce type de solution. II s'agit alors de vendre

\footnotetext{
${ }^{17}$ Alain Gerlache, publié le 7 juin 2012 sur le site belge RTBF, « Do No Track, l'antipub qui fâche les annonceurs » :

http://www.rtbf.be/info/chroniques/chronique do-not-track-l-anti-pub-qui-fache-les-annonceurs-alaingerlache?id=7782572
}

tic\&société - 6 (1), 2012 
Le ciblage comportemental,

une perte de contrôle des éditeurs sur les données de l'audience

du profil plutôt que la qualité d'une marque, d'un contenu ou une affinité. Seule une association des principaux sites de presse permettrait d'avoir une audience suffisamment significative pour réaliser le ciblage comportemental de façon avantageuse.

Le recueil d'informations sur une audience, qualifiée par son attirance pour les contenus médias, intéresse énormément les entreprises de ciblage comportemental, dans la mesure où il s'agit d'une "première sélection naturelle » d'un profil d'audience connu sociodémographiquement. Les éditeurs des sites médias sont donc la " cible du ciblage », car ils attirent des audiences qualifiées, permettant la constitution de profils valorisables pour les entreprises de ciblage comportemental. Le risque est cependant que ces internautes soient repérés sur les sites de presse pour être commercialisés en réseau sur d'autres sites web.

"Les agences démarchent les sites médias, le but étant de récolter la data, les données personnelles, les données de profiling, de façon à les utiliser ailleurs, d'où le problème. » (éditeur, CBS Interactive)

Le ciblage comportemental suppose une "brique technologique supplémentaire " entre les éditeurs et les annonceurs. Nous avons identifié une transformation profonde de l'activité publicitaire avec le passage d'un ciblage contextualisé aux côtés d'un contenu, dans un environnement, à un ciblage basé sur le profilage des utilisateurs par l'exploitation de leurs données personnelles. Les éditeurs doivent se positionner face à ce qui apparaît être une manifestation de cette transformation plus générale de la publicité en ligne :

" Toute la partie data-mining, ciblage comportemental, de toute façon, quel que soit ce qu'il en sorte, va nous obliger à considérer de plus en plus l'aspect exploitation des données, des profils, etc. Les gens payent avec leurs données personnelles. " (Éditeur, CBS Interactive)

Les entreprises de presse cherchent à collaborer avec ces acteurs pour ne pas rester en marge de la compétition. Cependant, elles prennent le risque de payer cher un service et une expertise technique qu'elles ne maîtrisent pas et qui pourrait leur échapper. À l'inverse, une attitude trop attentiste pourrait les 
conduire à être dépassées par une appropriation et une intégration de ces outils mieux réalisée par les acteurs de l'internet.

Ce qui apparaît le plus préjudiciable aux sites de presse est une réintermédiation de la relation avec leur audience. Perdre ce contact direct, c'est aussi se priver de la capacité à le valoriser soi-même et accepter de devoir s'en remettre à de nouveaux intermédiaires. La publicité en ligne élargit considérablement l'univers de concurrence pour les sites de presse. Les annonceurs ne passent plus de façon exclusive par les sites médias, mais aussi par d'autres sites tels que les portails et les moteurs de recherche. Ainsi, la relation qui les lie aux éditeurs se distend, en raison des alternatives existantes et des intermédiaires venant s'intégrer à la chaîne de valeur. En favorisant des modes d'achats alternatifs à l'achat direct sur un site média, les annonceurs expriment une exigence de mesurabilité, d'un suivi instantané et d'une comparabilité de la performance des espaces publicitaires, a contrario d'une mesure d'audience des contenus auxquels la publicité est indexée. Le ciblage publicitaire, autrefois lié au contexte de l'exposition et à l'environnement éditorial juxtaposé à la publicité, se transforme en ciblage basé sur les caractéristiques des individus, l'historique de leur navigation et de leur exposition à la publicité.

Pour les éditeurs, ces solutions représentent un risque double: se faire déposséder de la connaissance de leur audience et perdre la main sur le processus de qualification des espaces publicitaires. Globalement, l'expérience du ciblage comportemental pour les éditeurs et les régies publicitaires a été plutôt décevante. Elle n'a pas permis de faire augmenter significativement le prix des espaces publicitaires et reste un apport de revenu marginal. En somme, l'innovation technique telle qu'elle a été présentée aux régies publicitaires n'a pas tenu ses promesses. La difficulté pour les éditeurs est la perte d'un contact direct à l'audience. Dans une certaine mesure, la relation de confiance instaurée avec l'audience est minée par ces pratiques.

Les éditeurs se doivent d'être vigilants quant à l'accès aux données de leurs utilisateurs par ces nouveaux intermédiaires. Les données sont centrales pour le fonctionnement de ces plateformes publicitaires. Leur maîtrise devient un atout stratégique fort dans la relation-client avec les annonceurs. Le régulateur pourrait contraindre le développement du marché des données personnelles utilisées à des fins publicitaires. Ceci entraverait la dynamique d'évolution du marché publicitaire, en le privant d'outils et de solutions techniques en fort développement, mais rétablirait peut-être un certain équilibre de la relation marchande entre les éditeurs et les annonceurs.

L'efficacité du ciblage comportemental paraît très relative, puisque les prix de la publicité en ligne n'ont pas sensiblement progressé grâce à ces techniques. 
Le ciblage comportemental,

une perte de contrôle des éditeurs sur les données de l'audience

Cependant, elles dessinent une évolution du monde de la publicité en ligne, de plus en plus basée sur l'échange et le commerce de données liées aux utilisateurs.

\subsection{Les perspectives d'évolution de l'usage des données comportementales}

La plupart des investissements réalisés dans le secteur de la publicité en ligne, qu'il s'agisse de l'achat de réseaux et de serveurs publicitaires ou de solutions de ciblage comportemental, révèlent une croyance forte de l'industrie dans la possibilité d'un meilleur appariement publicitaire grâce aux technologies numériques (Evans, 2008, p.381). Les analystes de la publicité en ligne estiment qu'un usage croissant des données, déclarées ou inférées, des visiteurs de sites web sera réalisé. Ceci afin d'améliorer la personnalisation de l'exposition à la publicité et de la rendre plus efficace. Le président-directeur général de Weborama, Alain Levy déclarait fin mai 2012 à l'occasion du rachat d'une plateforme d'échange de données (data exchange), destinée à équiper l'achat de publicité sur internet, "pouvoir cibler des audiences et des segments de consommateurs, et non plus uniquement des emplacements publicitaires ॥. II s'agit, selon lui, d'un des enjeux clés du nouvel écosystème publicitaire qui se met en place sur le web ${ }^{18}$.

L'objectif ultime du ciblage publicitaire est d'adresser le bon message à la bonne personne au bon moment. Le moment est un facteur clé du ciblage publicitaire sur internet. II prend une importance grandissante, avec le système des places de marché publicitaires (ad exchanges), où est pratiqué le ciblage en temps réel de la publicité sous forme d'enchères (real time bidding).

Le ciblage comportemental propose un appariement qui transforme la logique de l'activité publicitaire en s'appuyant sur les données comportementales des utilisateurs, observées à partir des traces laissées par leur navigation. Le ciblage n'est pas une nouveauté pour le secteur publicitaire qui le pratique depuis plus de cinquante ans dans la publicité média et le marketing direct. Néanmoins, celui-ci atteint un degré de raffinement et de précision qui n'existait pas auparavant. Les technologies numériques permettent, en s'appuyant sur l'abondance de données créées par les usagers (volontairement ou non), d'offrir des possibilités de profilage et de segmentation

\footnotetext{
18 Journal du Net, article de Nicolas JAIMES, publié le 30 mai 2012, «Weborama rachète le data exchange Datvantage ",

http://www.journaldunet.com/ebusiness/publicite/weborama-datvantage-0512.shtml
} 
marketing dépassant largement les données sociodémographiques usuellement utilisées par l'industrie publicitaire dans le cadre de la publicité média. II n'est cependant pas évident que cette tendance à l'usage des données comportementales à des fins publicitaires affirme son efficacité. En effet, elle doit affronter aussi bien des difficultés intrinsèques - car la collecte et le traitement des données posent de nouveaux défis quant à la fiabilité des données obtenues et au caractère exploitable de celles-ci - qu'extrinsèques face aux réticences des consommateurs liées à la vie privée.

Jusqu'ici, les méthodes utilisées pour réaliser le ciblage de la publicité en ligne prenaient peu appui sur la puissance de calcul des technologies numériques. Le développement de solutions technologiques par de nouveaux intermédiaires vient accélérer l'amélioration des techniques de ciblage. En se centrant sur des données produites par la navigation des internautes, le ciblage comportemental participe à transformer la logique de fonctionnement de l'activité publicitaire.

La compétition dans la maîtrise des outils de la publicité en ligne joue en défaveur des éditeurs issus de la presse imprimée. Les grands acteurs de l'Internet ont des outils technologiques qui permettent aux annonceurs de mieux segmenter leur clientèle, de tirer parti des potentialités interactives de la publicité en ligne, tandis que les sites de presse disposent de contenus originaux susceptibles d'attirer des audiences qualitatives. Un terrain d'entente pourrait donc être trouvé entre ces différents types d'acteurs. Ainsi, les régies publicitaires des sites de presse s'appuieraient sur des outils technologiques plus perfectionnés, séduisants pour les annonceurs et parviendraient dans le même temps à maintenir un niveau de prix élevé pour leurs emplacements publicitaires. Des formes de «coopétition » devraient donc être envisagées (Smyrnaios, Rebillard, 2010).

Notre enquête s'inscrit dans le contexte de crise du marché publicitaire, liée à la crise économique et financière mondiale de 2008. Nos résultats ont pu être influencés par la conjoncture économique, conduisant à une recherche de la " performance » et un repli du marché publicitaire vers le marketing direct plutôt que la publicité média. Toutefois, nous observons un mouvement plus profond d'hybridation des logiques publicitaires média et hors-média sur internet, auquel est lié le développement des techniques de ciblage à partir des données des internautes. Notre hypothèse est qu'il s'agit d'une tendance plus structurelle de l'évolution du marché de la publicité en ligne. Notre enquête basée sur des entretiens qualitatifs illustre, à partir du ciblage comportemental, une dynamique d'innovation technique du marché publicitaire, basée sur la collecte et le traitement des données des internautes à des fins commerciales. II s'agit d'une extension de la chaîne de la valeur de la publicité en ligne, marquée par 
Le ciblage comportemental,

une perte de contrôle des éditeurs sur les données de l'audience

l'intégration d'acteurs issus d'une culture liée à l'ingénierie technique plutôt qu'à la production et à la diffusion de contenus aux côtés desquels est affichée une publicité.

L'extension des possibilités de ciblage à des formes spécifiques à l'Internet trouve une continuité et un prolongement avec la publicité sur mobile. L'interactivité n'est plus propre à l'Internet fixe, mais s'étend à un ensemble d'appareils connectés, tels que les tablettes et le mobile. De plus, le caractère personnel du smartphone rejoint et amplifie une tendance à la communication commerciale individualisée, c'est-à-dire dirigée vers un individu en fonction de ses caractéristiques particulières. Cependant, là aussi, le régulateur pourrait avoir son mot à dire. 


\section{Références bibliographiques}

ATTIAS D., 2008, "La presse sur Internet: quelles stratégies d'audience ? ", dans N. SONNAC et X. GREFFE (dir.), CultureWeb, pp. 513-531.

BOYD D. et K. CRAWFORD, 2011, "Six provocations for Big Data", paper presented at Oxford Internet Institute's "A Decade in Internet Time: Symposium on the Dynamics of the Internet and Society", 21 septembre, [http://papers.ssrn.com/sol3/Delivery.cfm/SSRN ID1926431 code1210838.p df?abstractid=1926431\&mirid=1, consulté le 15 juin 2012.]

CHANG-HOAN C. et J.C. HONGSIK, 2004, "Why do people avoid advertising on the internet?", Journal of Advertising, Vol. 33, n 4 Hiver, pp. 89-97

DREZE X. et F.-X. HUSSHERR, 2003, "Internet advertising: Is anybody Watching?", Journal of Interactive Marketing, Vol. 17, n4, Autumn (Fall), pp. 8-23.

EVANS D. S., 2008, "The Economics of the Online Advertising Industry", Review of Networks Economics, vol. 7, n 3, pp. 359-391.

EVANS D. S., 2009, "The Online Advertising Industry: Economics, Evolution, and Privacy", Journal of Economic Perspectives, vol. 23, n 3, pp. 37-60

GABSZEWICZ J. et N. SONNAC, 2010 (2006), L'industrie des médias à l'ère du numérique, Paris, Éditions La Découverte.

GOLDFARB A. et C. E. TUCKER, 2010, "Privacy Regulation and Online Advertising", Working papers series,

[http://papers.ssrn.com/sol3/papers.cfm?abstract id=1600259, consulté le 15 juin 2012.]

GOLDFARB A. et C. E. TUCKER, 2011, "How do advertising standards affect online advertising?", Working paper, [http://server1.tepper.cmu.edu/Seminars/docs/SSRN-id1745645.pdf, consulté le 15 juin 2012]

GOLDFARB A. and C. E. TUCKER, 2010, "Online Display Advertising: Targeting and Obtrusiveness", Marketing Science, Vol. 30, n 3, May-June, pp. 389-404.

HOLLIS N., 2005, "Ten Years of learning How Online Advertising Builds Brands", Journal of Advertising Research, vol. 45, n 2, pp. 255-269. 
Le ciblage comportemental,

une perte de contrôle des éditeurs sur les données de l'audience

NAPOLI P. M., 2003, Audience Economics : media institutions and the audience marketplace, New-York, Columbia University Press.

IYER G., D. SOBERMAN and J.M. VILLAS-BOAS, 2005, "The targeting of advertising", Marketing Science, Vol. 24, n 3 Été, pp. 461-476.

JOHNSON J.P., 2009, "Targeted advertising and advertising avoidance", Working paper, [http://www.wcas.northwestern.edu/csio/Conferences/DUCSIO-T-2009/Johnson.pdf, consulté le 15 juin 2012]

KESSOUS E., K. MELLET et M. ZOUINAR, 2010, "L'économie de l'attention : Entre protection des ressources cognitives et extraction de la valeur », Sociologies du travail, $n^{\circ} 3$, pp. 359-373.

OUAKRAT A., J. S. BEUSCART et K. MELLET, 2010, « Les régies publicitaires de la presse en ligne ", Réseaux, vol. 160-161, n²/3, pp. 134-161.

OUAKRAT A., 2011, La publicité en ligne sur les sites de presse. Construction du marché, logiques de fonctionnement et perspectives d'évolution, Thèse de Doctorat, Université Panthéon-Assas Paris 2.

PORTER M., 1986, L'avantage concurrentiel, Paris, InterEditions.

REBILLARD F. et N. SMYRNAIOS, 2010, "Les infomédiaires, au cœur de la filière de l'information en ligne », Réseaux, vol. 160-161, n 2/3, pp. 163-194.

TUROW J., 2012, The Daily You: How the Advertising Industry is Defining Your Identity and Your Worth, Yale University Press, 288 p.

TUROW J., 2012, "A guide to the Digital Advertising Industry That's Watching Your Every Click", The Atlantic (Boston), February 7, [http://www.theatlantic.com/technology/archive/2012/02/a-guide-to-the-digitaladvertising-industry-thats-watching-your-every-click/252667/, consulté le 15 juin 2012]

VAN COUVERING E., 2008, "The History of the Internet Search Engine: Navigational Media and the Traffic Commodity", Information Science and Knowledge Management, Vol. 14, n³, pp. 177-206.

\section{Rapports}

IDATE, 2010, « Stratégies des régies en ligne et modalités de valorisation de la publicité sur Internet », Rapport IDATE pour la DDM, Fontaine Gilles, Sophie Girieud, Florence Le Borgne, Tiffany Sauquet, 90055, Janvier, V3, 53 pages, 


\section{Alan OUAKRAT}

[www.ddm.gouv.fr/IMG/pdf/Rapport Final Site DGMIC 2010-02.pdf, consulté le 15 juin 2012]

SmartAdServer, 2009, Communiqué de presse, Smart AdMetrics, baromètre performances bannières, septembre :

[http://www.smartadserver.com/smart/miseajour/cp 072009/Doc1.pdf, consulté le 15 juin 2012] 